\title{
Diacronie
}

Studi di Storia Contemporanea

$\mathrm{N}^{\circ} 21,1$ | 2015

Le città di Babele

\section{Santo Peli, Storie di Gap. Terrorismo urbano e \\ Resistenza}

Andrea Martini

\section{(2) OpenEdition}

Journals

Edizione digitale

URL: http://journals.openedition.org/diacronie/1928

DOI: 10.4000/diacronie. 1928

ISSN: 2038-0925

Editore

Association culturelle Diacronie

Notizia bibliografica digitale

Andrea Martini, «Santo Peli, Storie di Gap. Terrorismo urbano e Resistenza », Diacronie [Online], № 21, 1 । 2015, documento 9, Messo online il 01 mars 2015, consultato il 24 septembre 2020. URL : http:// journals.openedition.org/diacronie/1928; DOI : https://doi.org/10.4000/diacronie.1928 


\section{Diacronie}

\section{RECENSIONE:}

\section{Santo PELI, Storie di Gap. Terrorismo urbano e Resistenza, Torino, Einaudi, 2014, 279 pp.}

a cura di Andrea MARTINI *

Nel libro intitolato La Resistenza in Italia del 2004, Santo Peli lamentava lo scarso progresso degli studi a proposito dei Gap (Gruppi di azione patriottica): «Le informazioni che utilizziamo su struttura, funzionamento e azioni dei Gap coincidono in gran parte con ciò che ne hanno scritto gli stessi organizzatori e dirigenti comunisti [...] mentre mancano ricerche originali ed esaustive basate sull'analisi critica delle fonti dirette»1. Rimarcava inoltre la mancanza di dati analitici concernenti i Gap, quali il numero dei gappisti, quello dei caduti, le varie fasi di queste formazioni e la loro distribuzione territoriale ${ }^{2}$. A distanza di dieci anni da quel volume, l'autore, che si è più volte confrontato con la Resistenza italiana e con le sue criticità ${ }^{3}$, tenta di colmare questa lacuna nel suo ultimo lavoro, Storie di Gap.

Conclusioni affrettate e rappresentazioni stereotipate dei gappisti, prodotte nel corso degli anni, hanno finito per fornire una visione alquanto deformata dei Gap. Il tentativo di Santo Peli è dunque quello di ricostruirne genesi e sviluppo e di sciogliere

\footnotetext{
${ }^{1}$ PELI, Santo, La Resistenza in Italia. Storia e critica, Torino, Einaudi, 2004, p. 259.

${ }^{2}$ Alcuni studi parziali sui Gap con cui Peli si è confrontato sono: GIOVANA, Mario, I gruppi di azione patriottica: caratteri e sviluppi di uno strumento di guerriglia partigiana, in POGGIO, Pier Paolo, MICHELETTI, Bruno (a cura di), «La guerra partigiana in Italia e in Europa», in Annali della Fondazione Luigi Micheletti, 5, 1998, pp. 201-215; SANDRI, Renato, Gruppi di azione patriottica, in COLLOTTI, Enzo, SANDRI, Renato, SESSI, Frediano (a cura di), Dizionario della Resistenza, Vol. II, Torino, Einaudi, 2001, pp. 209, 213.

3 Oltre all'opera sopra citata si vedano: PELI, Santo, Storia della resistenza italiana, Torino, Einaudi, 2006; ID., "Alcune idee sullo stato degli studi sulla Resistenza in Italia», in Italia contemporanea, 255, 2009, pp. 244-249; ID., «La violenza nella Resistenza italiana», in Terra d'Este, 38, 2009, pp. 21-31. L'autore si era già confrontato con i Gap nel suo studio ID., I Gruppi di azione patriottica, in ISNENGHI, Mario (a cura di), Gli italiani in guerra. Conflitti, identità, memorie dal Risorgimento ai nostri giorni, vol. III, tomo II, Torino, Utet, 2008, pp. 369-375.
} 
alcuni nodi interpretativi per oltrepassare le «deprecazioni calunniose» e le «acritiche esaltazioni» di cui a lungo le formazioni gappiste hanno risentito4.

L'obiettivo di Santo Peli è ambizioso, ma è frenato da alcune difficoltà oggettive: la scarsa presenza di documenti archivistici dovuta alla natura clandestina dei Gap, le criticità tipiche delle ricostruzioni e relazioni ufficiali di partito di cui disponiamo e, infine, la frammentarietà delle vicende gappiste. Questo persuade l'autore a declinare il titolo al plurale, Storie di Gap appunto, e ad esaminare solo alcune formazioni ritenute emblematiche, in particolare quella genovese, torinese e milanese al Nord, e quella fiorentina e romana al Centro, riservando un capitolo a parte al gappismo emiliano. Sebbene le questioni toccate da Peli siano numerose, ci limitiamo ad esporre quelle che, a nostro giudizio, rappresentano gli assi portanti dell'intero volume, cioè il ruolo assegnato ai Gap dal Partito comunista e i rapporti con il Pci stesso, i dilemmi morali dei gappisti e il nesso tra le loro azioni e le rappresaglie nazifasciste, partendo proprio da quest'ultimo aspetto.

Secondo Pietro Secchia è inverosimile pensare che fossero le gesta gappiste a causare le reazioni dell'occupante tedesco, anzi i Gap - a suo giudizio - ottennero l'effetto opposto: «al terrore occorreva reagire col terrore perché solo così, a lungo andare, i tedeschi avrebbero esitato, temendo a loro volta la rappresaglia partigiana, ad applicare i loro metodi scientificamente criminosi»5. Giorgio Bocca, al contrario, sosteneva che le azioni gappiste finissero per provocare il nemico, inasprendone l'occupazione in una sorta di «autolesionismo premeditato» ${ }^{6}$. Nel tentativo di evitare ogni banalizzazione della questione, Santo Peli non sposa nessuna delle due versioni, ritenendo esagerata ed encomiastica la prima, «assai schematica e riduttiva, oltre che tendenziosa»7 la seconda. Eppure, secondo l'autore, almeno in una prima fase, i gappisti e i vertici del Pci erano consapevoli che «per creare un clima di guerra, per costringerli a mostrare anche nelle città del Centronord il vero volto dell'occupazione, $\mathrm{i}$ tedeschi vanno attaccati, subito e duramente, e la rappresaglia è un elemento dolorosamente utile, che serve a bruciare gli spazi di mediazione, i tentennamenti» ${ }^{8}$. In altre parole - a suo avviso - in un primo momento i Gap, pur di turbare quel clima di torpore diffuso nelle grandi città dove l'occupazione tedesca mostrava il volto più tenue, erano disposti ad accettare, come effetto collaterale delle proprie gesta, le rappresaglie

\footnotetext{
4 PELI, Santo, Storie di Gap. Terrorismo urbano e Resistenza, Torino, Einaudi, 2014, p. 9. 5 SECCHIA, Pietro, FRASSATI, Filippo, Storia della Resistenza. La guerra di liberazione in Italia 1943-1945, Roma, Editori Riuniti, 1965, p. 392.

6 BOCCA, Giorgio, Storia dell'Italia partigiana, Roma-Bari, Laterza, 1967, p. 135.

7 PELI, Santo, Storie di Gap, cit., p. 23.

8 Ibidem.
} 
nemiche che avrebbero scosso i cittadini, imponendo loro di scegliere da che parte stare. Del resto Giorgio Amendola in una sua memoria, tenne a precisare che «il problema delle rappresaglie era stato posto e risolto una volta per sempre allinizio della guerra partigiana, in Italia, come prima in Francia e negli altri paesi occupati dai nazisti. Accettare il ricatto delle rappresaglie voleva dire rinunciare in partenza alla lotta»9. Ciò non significa, tuttavia, che i gappisti agissero in modo scriteriato e senza alcuna coscienza delle conseguenze delle proprie azioni: sapevano, che le vittime di eventuali rappresaglie sarebbero stati civili innocenti, ma soprattutto compagni antifascisti, se non persino amici. Infatti «in ambito urbano - precisa Peli - le principali vittime della strategia terroristica nazifascista sono i detenuti politici prelevati dalle carceri» ${ }^{10}$. Si tratta dunque di scelte difficili che laceravano gli animi dei gappisti.

Inevitabilmente il nesso tra azione partigiana e rappresaglia nazifascista rimanda al dilemma morale che attanaglia ogni singolo gappista, dunque al secondo nodo interpretativo caratterizzante la ricerca di Peli. Innanzitutto l'autore evidenzia la diversità tra la vita del gappista e quella del partigiano di montagna. L'atto di uccidere, ad esempio, assume una fisionomia differente: mentre il partigiano conduce un'azione di gruppo e si ritrova a metabolizzare l'assassinio insieme ai suoi compagni, il gappista agisce da solo o attorniato da pochi uomini, spara al proprio obiettivo a distanza ravvicinata, scorgendone il volto. Non è un caso che i gappisti non sempre trovassero la forza di portare a termine l'azione, o che molti, nel bel mezzo dell'attentato o immediatamente prima, rinunciassero ad agire. Le testimonianze dirette o indirette cui ricorre Santo Peli, sono numerose. Emblematico è il ricordo lasciatoci da Amendola di un suo compagno: «Vidi un compagno (non importa il nome) tornare tutto avvilito da un'azione: era arrivato vicino a un tedesco, il viale era vuoto, si poteva sparare senza eccessivi pericoli, ma all'ultimo momento il dito si era fermato sul grilletto. E' difficile mi disse - uccidere a sangue freddo un uomo che non si conosce» ${ }^{11}$. Alcuni abbandonarono la città perché provati da una lotta di questo tipo. Anche il gesto di far esplodere una dinamite in un bordello frequentato notoriamente dai nazifascisti piuttosto che in un ristorante bazzicato dagli occupanti tedeschi, sebbene risultasse apparentemente più facile che premere un grilletto a pochi metri dall'obiettivo, sollevava forte dubbi etici nell'animo del gappista, perché il rischio che innocenti, come il personale che vi lavorava, potessero morire era sempre elevato.

9 AMENDOLA, Giorgio, Lettere a Milano, Roma, Editori Riuniti, 1973, p. 293.

1o PELI, Santo, Storie di Gap, cit., p. 261.

${ }^{11}$ AMENDOLA, Giorgio, op. cit., p. 225. 
L'importanza accreditata dall'autore alla questione morale che attanagliava i gappisti è funzionale al suo desiderio di smitizzarli, frantumando le ricostruzioni a posteriori dei vertici comunisti propensi a descrivere gli aderenti ai Gap come figure eroiche che non crollavano neppure sotto tortura. Nel pantheon del Partito erano esclusi coloro che cedevano alle sevizie: per acquisire il riconoscimento di gappista occorreva aderire in pieno alla «mistica del martirio»12. Peli elogia gli uomini che sopravvissero alle violenze nazifasciste; è indubbio che essi siano da commemorare, ma è inimmaginabile pensare che «il loro esempio fosse alla portata della maggioranza dei gappisti» ${ }^{13}$.

Dalle pagine di Storie di Gap si comprende quanto difficile sia stato il rapporto tra le formazione gappiste e il Pci; questa complessa relazione, per altro, rappresenta a nostro giudizio la chiave interpretativa più rilevante per comprendere la storia dei Gap.

Il Partito Comunista, da cui i Gap dipendevano, accreditava ai gruppi di azione patriottica un ruolo fondamentale: questi avrebbero dovuto innescare una guerra di massa contro l'occupante fascista. Per realizzare quest'obiettivo, occorreva reclutare unicamente comunisti, quindi operai, disposti ad agire in clandestinità. Nonostante le speranze del partito, il numero di gappisti rimase sempre limitato; con l'eccezione di Milano, dove le adesioni furono inizialmente una dozzina, a Torino, Genova, Bologna, Rimini, Firenze e Roma l'arruolamento procedette a stento. Del resto, era improbo trovare uomini disposti a sposare la causa terroristica, sia per ragioni morali che per difficoltà materiali, dovendo abbandonare lavoro e affetti. Inoltre, i mezzi a disposizione del partito erano pochi: mancavano i luoghi dove nascondersi, le armi erano precarie e i soldi a disposizione scarseggiavano. Se consideriamo questa carenza di risorse, le prime gesta gappiste stupiscono ancora di più. L'uccisione di Gino Gobbi a Firenze l'1 dicembre 1943, quella di Aldo Resega il 18 dicembre 1943 a Milano, l'attentato al Colonnello Italo Ingaramo il 29 aprile 1944 che aveva insediato, protetto e difeso a Firenze il maggiore Mario Carità e, infine, il tentato assassinio del questore di Milano, Camillo Nicolini Santamaria del 3 febbraio 1944 furono azioni sorprendenti ed efficaci. L'autore, tuttavia, rileva come successi così lampanti fossero la premessa a momenti di crisi. Questi erano in parte inevitabili: i cordoni della sicurezza si tesero e, al contempo, i tedeschi abbandonarono rapidamente la strategia moderata che ne aveva contrassegnato la prima fase dell'occupazione delle grandi città; anche i gappisti, però, commisero degli errori che ne facilitarono l'arresto: tornavano, ad esempio, sul luogo dell'attentato per comprenderne l'impatto e si ritrovavano in luoghi pubblici per

12 PELI, Santo, Storie di Gap, cit., p. 214.

13 Ibidem, p. 228. 
progettare nuove imprese. L'attacco gappista condotto alla casa del fascio di Sesto San Giovanni (Milano) il 10 febbraio 1944 evidenzia tutta l'ingenuità di queste formazioni: «colpire nella zona dove i gappisti sono, alla lettera, di casa, sembra contrario a ogni ragionevole strategia che si basi sulla clandestinità, sull'ubiquità, sull'invisibilità»14, ricorda Santo Peli, eppure, sulla scia degli iniziali successi, il Gap milanese decideva comunque di effettuare l'attentato. Questo provocò la morte di quattro fascisti, ma la reazione delle forze dell'ordine fu immediata. Catturarono per primo l'uomo infiltratosi presso la Casa del Fascio che aveva tardato ad abbandonare la città; costui, una volta intercettato, rivelò informazioni compromettenti sui suoi compagni che abitavano a Sesto San Giovanni in luoghi familiari ai fascisti. Al pari di Milano, i gappisti torinesi, genovesi, fiorentini e romani si sciolsero per motivi analoghi.

I vertici comunisti non risparmiavano critiche severe ai Gap; a detta del partito, $\mathrm{i}$ gappisti non avrebbero dovuto conoscersi, abitare negli stessi locali, a maggior ragione se già perquisiti dalle forze dell'ordine ${ }^{15}$. Santo Peli si interroga però sullo spessore di queste accuse. In primo luogo, sottolinea come la «perdurante compenetrazione, una ininterrotta osmosi tra i combattenti clandestini e l'habitat da cui provengono» ${ }^{16}$ sia stata benefica nella prima fase di vita dei responsabili del "lavoro sportivo" ${ }^{17}$. E' altrettanto vero che «il permanere dei gappisti nel quartiere dove sono nati e cresciuti, o il protrarsi di vecchie abitudini e di usuali frequentazioni, è la principale causa delle catture ${ }^{18}$. La questione è complessa dunque, ma sorge spontaneo l'interrogativo se i gappisti potessero agire diversamente. Secondo l'autore un atteggiamento più ortodosso si sarebbe potuto realizzare se il Pci avesse dispiegato aiuti materiali maggiori. Era troppo facile - a suo giudizio - rovesciare ogni responsabilità sui singoli terroristi. Tale comportamento comunista svolge

una funzione autoassolutoria, visto che sia la selezione delle persone da immettere, sia il reperimento dei mezzi indispensabili alla clandestinità, dipendono dai Comitati militari delle federazioni, in ultima istanza dal Partito. Lamentare il mancato rispetto delle norme della clandestinità, senza averne creato i presupposti,

\footnotetext{
14 Ibidem, p. 98.

${ }_{15}$ È il caso ad esempio di Mario Fiorentini, gappista romano. Egli risiede nelle vicinanze di via Rasella, e vi abita nonostante sia sfuggito fortunosamente a tre retate tedesche, perché lì soggiorna Lucia, la sua compagna, che aveva problemi di salute. Cfr. PELI, Santo, Storie di Gap, cit., p. 242.

${ }^{16}$ PELI, Santo, Storie di Gap, cit., p. 238.

${ }^{17}$ L'espressione adoperata da Peli come sinonimo di Gap è piuttosto diffusa nei documenti interni al Partito comunista.

${ }_{18}$ PELI, Santo, Storie di Gap, cit., p. 238.
} 
è una consuetudine piuttosto diffusa nelle analisi critiche di cui le relazioni ufficiali abbondano ${ }^{19}$.

A suscitare contrasti vi erano anche le modalità d'azione dei Gap: a tale riguardo i vertici del partito non vantavano una visione unitaria. Pietro Secchia, ad esempio, il 7 dicembre 1944 ammoniva i Gap torinesi, invitandoli a non ricorrere ad azioni dinamitarde che uccidevano o ferivano innocenti, con il rischio ultimo di perdere il consenso popolare. Arturo Colombi, responsabile della federazione comunista di Torino, dovette obbedire, ma manifestò il suo disaccordo: precisò che si era in guerra e che i propri uomini si sforzavano di ridurre al minimo gli effetti collaterali delle proprie azioni. Appurate le difficoltà connaturate nei Gap, il Partito decise di affiancarvi le Sap (Squadre d'azione patriottica) che avrebbero dovuto proteggere la classe operaia e diffondere la ribellione in città. È però difficile accertare se e quanto le Sap colmarono le lacune dei Gap: in realtà l'autore constata una sorta di progressivo intreccio tra queste due formazioni. È senz'altro vero comunque che i gappisti svolsero nella Resistenza un ruolo maggiore nella prima fase piuttosto che nell'insurrezione finale, principalmente coordinata dalle Sap.

In conclusione, è complesso stabilire se in questo «corpo a corpo» ${ }^{20}$ con i nodi politici ed etici contrassegnanti l'esistenza dei Gap, Peli abbia prevalso. Senz'altro la carenza di studi locali, quanto mai auspicabili, e di fonti documentarie non lo hanno aiutato, ma non vi è dubbio che lo storico abbia colmato una lacuna sul panorama storiografico e abbia finito per riconoscere il giusto valore alle azioni terroristiche di alcuni uomini dai valori antifascisti. In altre parole ha spezzato l'anatema che si era prodotto nei confronti del termine terrorismo, concepito a lungo come «sinonimo di follia omicida senza giustificazioni» ${ }^{21}$.

\footnotetext{
19 Ibidem, p. 247.

${ }^{20}$ Ibidem, p. 9.

${ }^{21}$ Ibidem, p. 8.
} 


\section{* L'autore}

Andrea Martini è dottorando presso "L'Orientale" di Napoli dove sta conducendo una ricerca sulle Corti d'Assise straordinarie in Italia e sulla giustizia di transizione. Si è laureato in scienze storiche presso l'università di Padova con una tesi intitolata Processo alle fasciste. Le collaborazioniste in alcune provincie venete.

URL: < http://www.studistorici.com/progett/autori/\#Martini >

\section{Per citare questo articolo:}

MARTINI, Andrea, «Recensione: Santo PELI, Storie di Gap. Terrorismo urbano e Resistenza, Torino, Einaudi, 2014, 279 pp.», Diacronie. Studi di Storia Contemporanea : Le città di Babele, 29/3/2015,

URL:<http://www.studistorici.com/2015/03/29/martini_numero_21/ >

Diacronie Studi di Storia Contemporanea $\sqrt{3}$ www.diacronie.it

Risorsa digitale indipendente a carattere storiografico. Uscita trimestrale. redazione.diacronie@hotmail.it

Comitato di redazione: Jacopo Bassi - Luca Bufarale - Elisa Grandi - Deborah Paci - Fausto Pietrancosta - Matteo Tomasoni - Luca Zuccolo

Diritti: gli articoli di Diacronie. Studi di Storia Contemporanea sono pubblicati sotto licenza Creative Commons 2.5 Possono essere riprodotti a patto di non modificarne i contenuti e di non usarli per fini commerciali. La citazione di estratti è comunque sempre autorizzata, nei limiti previsti dalla legge. 